\title{
Associations of body shapes with insulin resistance and cardiometabolic risk in middle-aged and elderly Chinese
}

Yulin Zhou ${ }^{1,2+}$, Yanan Hou ${ }^{1,2+}$, Jiali Xiang ${ }^{1,2}$, Huajie Dai ${ }^{1,2}$, Mian Li ${ }^{1,2}$, Tiange Wang ${ }^{1,2}$, Shuangyuan Wang ${ }^{1,2}$, Hong Lin ${ }^{1,2}$, Jieli Lu ${ }^{1,2}, Y u X u^{1,2}$, Yuhong Chen ${ }^{1,2}$, Weiqing Wang ${ }^{1,2}$, Yufang Bi ${ }^{1,2}$, Min Xu $u^{1,2^{*+}}$ and Zhiyun Zhao ${ }^{1,2^{*+}}$

\begin{abstract}
Background: We aimed to define refined body shapes by using multiple anthropometric traits that represent fat distribution, and evaluate their associations with risk of insulin resistance (IR) and cardiometabolic disorders in a Chinese population.

Methods: We performed a cross-sectional analysis in 6570 community-based participants aged $\geq 40$ years. Four body circumferences (neck, waist, hip, and thigh) and their ratios were put simultaneously into an open-source Waikato Environment for Knowledge Analysis platform to select the worthiest indicators in determining IR. The ratio of the top 3 fat distribution indicators was used to define the refined body shapes.
\end{abstract}

Results: We defined 8 distinct body shapes based on sex-specific combinations of waist-to-hip ratio (WHR), waist-tothigh ratio (WTR), and waist-to-neck ratio (WNR), which differed in participants' distribution and risk of IR and related cardiometabolic disorders. In women, as compared to the low WHR-low WTR-low WNR shape, all body shapes were significantly associated with IR and related cardiometabolic disorders; while in men, the low WHR-high WTR-high WNR shape and the higher WHR related shapes were significantly associated with IR and related cardiometabolic disorders. Stratified by WHR, the results were consistent in women; however, no significant associations were detected in men.

Conclusions: We defined 8 distinct body shapes by taking WHR, WTR, and WNR, simultaneously into account, which differed in association with the risk of IR and related cardiometabolic disorders in women. This study suggests that body shapes defined by multiple anthropometric traits could provide a useful, convenient, and easily available method for identifying cardiometabolic risk.

Keywords: Body shape, Anthropometric traits, Insulin resistance, Cardiometabolic disorders

*Correspondence: della.xumin@163.com; zzybrad@hotmail.com

†Yulin Zhou and Yanan Hou contributed equally to the work

${ }^{\dagger}$ Min Xu and Zhiyun Zhao co-supervised the work

${ }^{1}$ Department of Endocrine and Metabolic Diseases, Shanghai Institute of Endocrine and Metabolic Diseases, Ruijin Hospital, Shanghai Jiao Tong University School of Medicine, 197 Ruijin 2nd Road, 200025 Shanghai, China

Full list of author information is available at the end of the article

\section{Background}

Obesity is a global public health concern. More than 1 billion adults suffer from overweight, and 650 million adults and 124 million adolescents suffer from obesity worldwide [1]. Obesity and related cardiometabolic disorders, such as insulin resistance, metabolic syndrome, hyperlipidemia, etc., are responsible for type 2 diabetes, cancers, cardiovascular diseases and related mortality [2-4]. To better identify and define obesity is extremely original author(s) and the source, provide a link to the Creative Commons licence, and indicate if changes were made. The images or other third party material in this article are included in the article's Creative Commons licence, unless indicated otherwise in a credit line to the material. If material is not included in the article's Creative Commons licence and your intended use is not permitted by statutory regulation or exceeds the permitted use, you will need to obtain permission directly from the copyright holder. To view a copy of this licence, visit http://creativecommons.org/licenses/by/4.0/. The Creative Commons Public Domain Dedication waiver (http://creativeco mmons.org/publicdomain/zero/1.0/) applies to the data made available in this article, unless otherwise stated in a credit line to the data. 
essential for the prevention and management of comorbidity and mortality.

Anthropometric traits such as body mass index (BMI), waist circumference, and waist-to-hip ratio (WHR) were widely used to classify obesity and body shapes (the apple or pear type), which plays a vital role in evaluating cardiometabolic risk [5-7]. However, these metrics cannot adequately discriminate variation in fat distribution and cannot adequately evaluate individual cardiometabolic risk. In clinical practice, individuals with the same BMI or waist circumference may have different health conditions. Some obese individual has been considered as "metabolically healthy obese [8], and some individuals in a normal range of BMI, still at increased risk of type 2 diabetes [9], suggesting that other factors, including other ectopic fat depots, may have a contribution [10].

Integrating multiple anthropometric information aids in defining a more accurate cardiometabolic risk [11-15]. For example, when incorporated calf circumference into the definition of metabolic syndrome, it demonstrated a higher cardiovascular and all-cause mortality risk than the traditional definition of metabolic syndrome alone [14]. Most indices of central fatness, including waist circumference, WHR, body adiposity index and a body shape index, were positively associated with a higher all-cause mortality risk independent of overall adiposity, which indicated measures of central adiposity could be used as a supplementary approach, in combination with BMI, to determine the risk of premature death [15]. Upper body subcutaneous fat is a distinct fat depot separate from abdominal subcutaneous fat that may confer increased cardiometabolic risk [16]. Neck circumference, as an indirect measure of upper body subcutaneous fat, is associated with cardiometabolic risk factors and subclinical atherosclerosis independent of BMI [16]. These previous studies suggested novel anthropometric traits play a vital role in predicting cardiometabolic risk.

In addition, it's uncertain whether the combination of multiple anthropometric traits may identify additional risk assessment of cardiometabolic disorders. Therefore, our study aimed, firstly, to define body shapes in a sexspecific manner by using several body circumferences or their ratio; secondly, to evaluate their associations with risk of insulin resistance and several major cardiometabolic disorders. The study will provide refined body shapes and further stratify individuals into different stratification of cardiometabolic risk based on these distinct body shapes.

\section{Methods}

\section{Study population}

The current cross-sectional analysis was based on one of the follow-up circles of our previous community-based cohort studies [17, 18]. Briefly, between August 2014 and May 2015, 6570 registered permanent residents aged $\geq 40$ years from Jiading district, Shanghai, China, participated in the health examination aimed to explore the effects of risk factors on type 2 diabetes and related chronic diseases. Participants missing data on anthropometric traits including height, weight, waist circumference, hip circumference, thigh circumference and neck circumference $(n=167)$, or missing data on biochemical measurements including systolic and diastolic blood pressure, fasting and $2 \mathrm{~h}$-OGTT plasma glucose, fasting serum insulin, total cholesterol, triglycerides, high-density lipoprotein cholesterol and low-density lipoprotein cholesterol $(n=163)$ were excluded. Finally, 6240 participants were included in the current analysis.

The study protocol was approved by the Institutional Review Board of Ruijin Hospital affiliated to Shanghai Jiao Tong University School of Medicine. All participants consented to the study and signed informed consent.

\section{Anthropometric measurements}

A standard questionnaire was used to collect information on sociodemographic characteristics, chronic diseases and medical history, physical activity and lifestyle factors (e.g. smoking and alcohol status). If participants consumed any kinds of cigarettes or alcohol regularly in the past 6 months, the current smoking or drinking status was defined as 'yes'. Physical activity in terms of MET hour/week was acquired and calculated according to the short form of the International Physical Activity Questionnaire (IPAQ) [19].

Anthropometric traits including height, weight, neck circumference, waist circumference, hip circumference and thigh circumference were measured by well-trained physicians according to a standard protocol. BMI was calculated as body weight in kilograms divided by body height squared in meters $\left(\mathrm{kg} / \mathrm{m}^{2}\right)$. Waist circumference was measured at the level of the umbilicus with the patient in the standing position. Hip circumference was measured at the tip of the bone around the greater trochanter of the femur. Thigh circumference was measured on the right leg directly below the gluteal fold [20]. Neck circumference was measured in the midway of the neck, below the laryngeal prominence [21]. Systolic and diastolic blood pressure were measured at the non-dominant arm with an automated electronic device (OMRON Model HEM-752 FUZZY, Omron Company, Dalian, China) three times consecutively with a 1-min interval after at least $10-$ min rest in the seated position. The average value of the three measurements was used in our analysis. 


\section{Biochemical measurements}

All participants underwent standard 75-g oral glucose tolerance tests (OGTT) after overnight fasting of more than $10 \mathrm{~h}$. Fasting and $2 \mathrm{~h}$-OGTT plasma glucose concentrations were measured using the glucose oxidase method through an autoanalyzer (Modular P800; Roche, Basel, Switzerland). Fasting and 2 h-OGTT serum insulin concentrations, and serum lipid profiles including total cholesterol, triglycerides, high-density lipoprotein (HDL) cholesterol and low-density lipoprotein (LDL) cholesterol were measured using an electrochemiluminescence assay (Modular E170; Roche, Basel, Switzerland).

\section{Definitions}

Insulin resistance index (homeostasis model assessment of insulin resistance, HOMA-IR) was calculated as fasting serum insulin $(\mu \mathrm{IU} / \mathrm{mL}) \times$ fasting plasma glucose $(\mathrm{mmol} / \mathrm{L})$ / 22.5. Excluding individuals treated with insulin or hypoglycemic agents, insulin resistance was defined as HOMA-IR $\geq 2.61$, which was the highest quartile of HOMA-IR. Diabetes was defined as fasting plasma glucose $\geq 7.0 \mathrm{mmol} / \mathrm{L}$ or $2 \mathrm{~h}$-OGTT plasma glucose $\geq 11.1 \mathrm{mmol} / \mathrm{L}$ or use of antidiabetic agents. Metabolic syndrome was diagnosed based on the National Cholesterol Education Program Adult Treatment Panel III criteria [22]. Individuals with three or more of the following five components were diagnosed to have metabolic syndrome: (1) blood pressure $\geq 130 / 85 \mathrm{mmHg}$ or taking antihypertensive drugs; (2) waist circumference $\geq 88 \mathrm{~cm}$ for women ( $\geq 102 \mathrm{~cm}$ for men); (3) serum triglyceride level $\geq 1.7 \mathrm{mmol} / \mathrm{L}$; (4) serum HDL cholesterol level $<1.29 \mathrm{mmol} / \mathrm{L}$ for women ( $<1.04$ for men); (5) fasting plasma glucose level $\geq 6.1 \mathrm{mmol} / \mathrm{L}$ or confirmed diagnosis of diabetes.

\section{Definition of body shapes}

Automated feature selection was performed by using the information gain attribute ranking method on the opensource Waikato Environment for Knowledge Analysis platform [23]. Information gain ranking was used to evaluate the worth of each variable (usually the clinical indicator) by measuring the entropy gain to the outcome. The greater the information gain a clinical indicator has, the more important the indicator is in the classification process. The model was built with logistic regression analysis in a sex-specific manner. The regression coefficient of each significant variable was regarded as the contribution level (Additional file 1: Fig. S1A) [23].

In the present study, we used insulin resistance as the outcome to evaluate the potential dominant anthropometric traits in a sex-specific manner. Anthropometric traits including $\mathrm{BMI}, \mathrm{WHR}$, waist-to-thigh ratio
(WTR), waist-to-neck ratio (WNR), neck-to-thigh ratio, neck-to-hip ratio, thigh-to-hip ratio were put into the selection process. As a result, BMI ranked first for determining insulin resistance, followed by WHR, WTR, and WNR in both men and women (Additional file 1: Fig. 1B).

As expected, BMI ranked first for determining insulin resistance in our analysis. According to our study objective, we aimed to define body shapes by using several circumferences or their ratios to identify additional risk assessment. Therefore, we took the 3 following ratios, WHR, WTR, and WNR, simultaneously into account to define the body shapes in a sex-specific manner and evaluate their associations with risks of insulin resistance and cardiometabolic disorders.

We calculated the medians of these 3 traits in a sexspecifically manner. The corresponding median value for WHR was 0.87 in women and 0.91 in men, for WTR was 1.66 in women and 1.74 in men, and for WNR was 2.48 in women and 2.34 in men. We performed a receiver operating characteristic (ROC) scatter plot analysis to examine the best cut-off value of WHR, WTR and WNR in relation to insulin resistance. We found that the best cutoff value of these 3 metrics almost equal to the median values. According to the medians of WHR, WTR, and WNR, individuals were divided into the high- and lowlevel groups respectively. Based on the 3 derived ratios, WHR, WTR, and WNR, we defined 8 distinct body shapes (Fig. 1).

\section{Statistical analysis}

Data were summarized as means \pm standard deviation (SD) or median (inter-quartile ranges) for continuous variables, and numbers (percentages) for categorical variables. Multiple variable logistic regression analysis was performed to evaluate associations of body shapes with insulin resistance and cardiometabolic disorders in sexspecific samples. Odds ratios (ORs) and the corresponding 95\% confidence intervals (CI) were calculated. The adjustments included age (years), BMI $\left(\mathrm{kg} / \mathrm{m}^{2}\right)$, current smoking (yes or no), current drinking (yes or no) and physical activity (MET-h/wk).

To assess the added value of the defined body shape in predictive models, we included these body shapes in the models of predicting cardiometabolic risk. We calculated the difference with or without the defined body shape $(\Delta \mathrm{C}$ statistic), net reclassification improvement (NRI), and integrated discrimination improvement (IDI). The $\mathrm{C}$ statistic measures concordance between model-based risk estimates and observed events. NRI and IDI measure the incremental prognostic effect that a new biomarker will have when added to an existing prediction model. 
A

Women $(n=3961) \quad$ Men $(n=2279)$
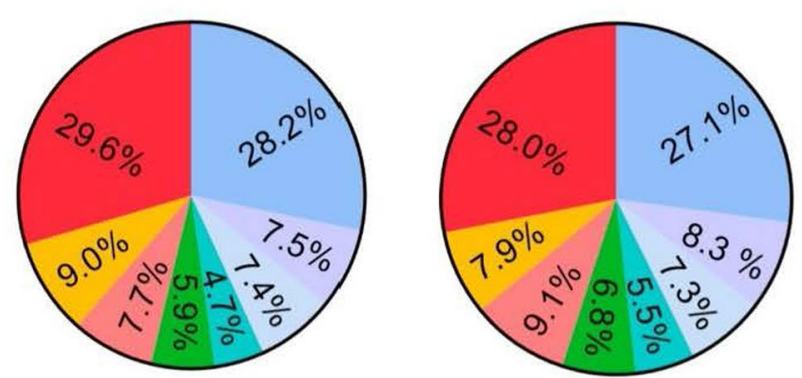

LOW WHR-IOW WTR-low WNR

Low WHR-low WTR-high WNR

Low WHR-high WTR-low WNR

Low WHR-high WTR-high WNR

High WHR-Iow WTR-low WNR

High WHR-low WTR-high WNR

High WHR-high WTR-low WNR

High WHR-high WTR-high WNR

B
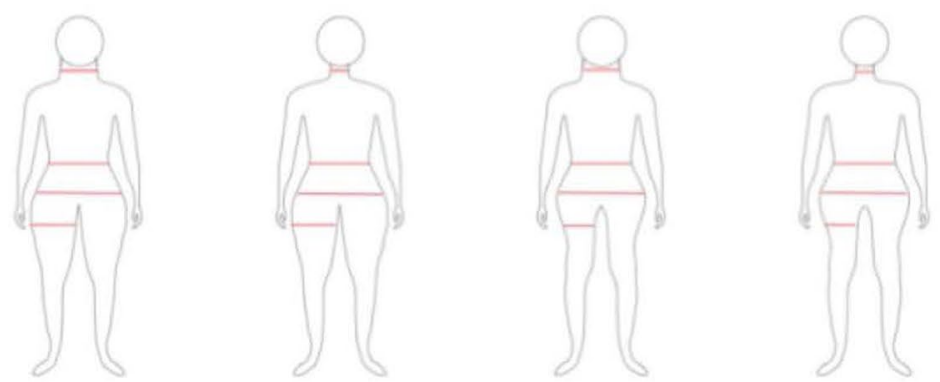

Low WHR- Low WHR- Low WHR- Low WHR-

Body shape low WTR- low WTR- high WTR- high WTR-

\begin{tabular}{lllll} 
& low WNR & high WNR & low WNR & high WNR \\
\hline Women, $\mathrm{n}(\%)$ & $1118(28.2)$ & $296(7.5)$ & $294(7.4)$ & $186(4.7)$ \\
Men, $\mathrm{n}(\%)$ & $618(27.1)$ & $189(8.3)$ & $167(7.3)$ & $125(5.5)$ \\
\hline
\end{tabular}

C

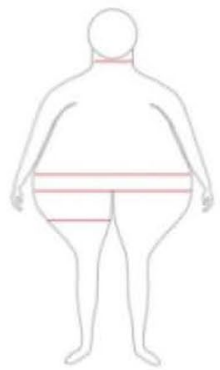

High WHR-

Body shape

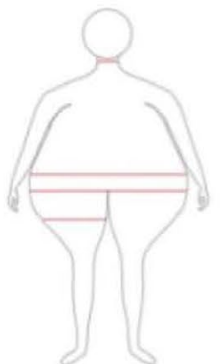

High WHR-

low WTR-

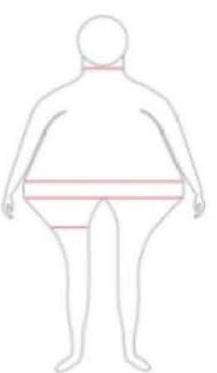

High WHR-

high WTR-

low WNR

$356(9.0)$

$180(7.9)$

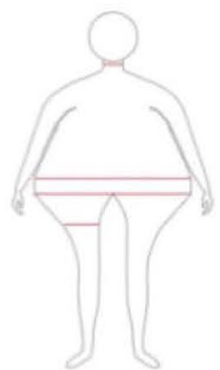

High WHR-

high WTR-

high WNR

$1172(29.6)$

Men, n (\%)

$154(6.8)$

$305(7.7)$

$639(28.0)$

Fig. 1 The distribution of body shapes in sex-specific groups. The individuals were divided into high- and low-level groups according to the medians of WHR, WTR, and WNR, respectively. Based on the 3 derived ratios, WHR, WTR, and WNR, we defined 8 distinct body shapes. The corresponding median value for WHR is 0.87 in women and 0.91 in men, for WTR is 1.66 in women and 1.74 in men, and for WNR is 2.48 in women and 2.34 in men. Abbreviation:WHR, waist-to-hip ratio; WTR, waist-to-thigh ratio; WNR, waist-to-neck ratio 
All the statistical analyses were performed with SAS version 9.4 (SAS Institute Inc, Cary, NC, USA). Statistical significance was set to a two-sided $P$ value $<0.05$.

\section{Results}

\section{Characteristics of study participants}

The present study including 3961 (63.4\%) women and $2279(36.3 \%)$ men with an average BMI was $25.0 \pm 5.9 \mathrm{~kg} /$ $\mathrm{m}^{2}$ for women and $25.1 \pm 3.5 \mathrm{~kg} / \mathrm{m}^{2}$ for men. Compared with men, women were younger, had lower level of several major anthropometric traits including neck circumference, waist circumference, hip circumference, thigh circumference, WHR, WTR, neck-to-hip ratio, and neckto-thigh ratio; higher level of HOMA-IR, systolic blood pressure, LDL- and HDL cholesterol and triglycerides (All $P<0.05$, Table 1).

Table 1 Characteristics of study participants in sex-specific samples

\begin{tabular}{|c|c|c|c|c|c|c|}
\hline & \multirow{2}{*}{$\begin{array}{l}\text { Total } \\
\text { / }\end{array}$} & \multicolumn{2}{|l|}{ Women } & \multicolumn{2}{|l|}{ Men } & \multirow[t]{2}{*}{$P$ value } \\
\hline & & l & Range & l & Range & \\
\hline$n(\%)$ & 6240 & $3961(63.4)$ & / & $2279(36.3)$ & / & / \\
\hline Age (years) & $62.2 \pm 8.74$ & $61.9 \pm 8.56$ & $44.3-91.2$ & $62.9 \pm 9.03$ & $40.1-94.9$ & $<0.0001$ \\
\hline Systolic blood pressure (mmHg) & $134.8 \pm 17.3$ & $135.1 \pm 17.7$ & $66.3-224.7$ & $134.2 \pm 16.5$ & $89.0-213.3$ & 0.03 \\
\hline Diastolic blood pressure $(\mathrm{mmHg})$ & $76.3 \pm 9.5$ & $75.4 \pm 9.5$ & $42.0-127.3$ & $77.8 \pm 9.7$ & $42.3-124.7$ & $<0.0001$ \\
\hline Current smoking, n (\%) & $1224(19.2)$ & $20(0.5)$ & / & $1204(51.5)$ & / & $<0.0001$ \\
\hline Current drinking, n (\%) & $900(14.1)$ & $45(1.11)$ & / & $855(36.5)$ & / & $<0.0001$ \\
\hline Physical activity (MET-h/wk) & $18(4.5-21.0)$ & $21.0(6.0-21.0)$ & $0-2380$ & $15.0(0.0-21.0)$ & $0-489$ & 0.60 \\
\hline \multicolumn{7}{|l|}{ Biochemical measurements } \\
\hline Fasting plasma glucose (mmol/L) & $6.15 \pm 1.47$ & $6.06 \pm 1.34$ & $1.40-20.8$ & $6.29 \pm 1.66$ & $4.21-20.0$ & $<0.0001$ \\
\hline HOMA-IR & $1.89(1.27-2.83)$ & $1.97(1.34-2.91)$ & $0.13-224$ & $1.74(1.13-2.61)$ & $0.19-226$ & $<0.0001$ \\
\hline Total cholesterol (mmol/L) & $5.95 \pm 0.96$ & $6.54 \pm 0.96$ & $2.44-19.3$ & $4.95 \pm 0.89$ & $2.68-8.42$ & 0.26 \\
\hline LDL cholesterol (mmol/L) & $3.67 \pm 4.13$ & $3.83 \pm 5.16$ & $1.51-10.9$ & $3.39 \pm 0.71$ & $1.14-6.07$ & $<0.0001$ \\
\hline HDL cholesterol (mmol/L) & $1.34 \pm 0.29$ & $1.39 \pm 0.30$ & $0.60-3.28$ & $1.25 \pm 0.29$ & $0.68-3.08$ & $<0.0001$ \\
\hline Triglycerides (mmol/L) & $1.51(1.09-2.14)$ & $1.55(1.12-2.19)$ & $0.45-46.2$ & $1.43(1.03-2.04)$ & $0.38-15.96$ & $<0.0001$ \\
\hline \multicolumn{7}{|l|}{ Anthropometric measurements } \\
\hline Body mass index $\left(\mathrm{kg} / \mathrm{m}^{2}\right)$ & $25.0 \pm 5.2$ & $25.0 \pm 5.9$ & $15.0-35.1$ & $25.1 \pm 3.5$ & $17.6-37.2$ & 0.41 \\
\hline Waist circumference $(\mathrm{cm})$ & $83.6 \pm 9.9$ & $81.8 \pm 10.0$ & $30.0-163.0$ & $86.7 \pm 9.8$ & $35.0-164.0$ & $<0.0001$ \\
\hline Neck circumference $(\mathrm{cm})$ & $34.4 \pm 3.2$ & $33.0 \pm 3.2$ & 18.4-79.0 & $36.9 \pm 3.3$ & $25.2-70.0$ & $<0.0001$ \\
\hline Hip circumference (cm) & $93.9 \pm 7.7$ & $93.2 \pm 8.0$ & $25.0-201.0$ & $95.0 \pm 7.1$ & $29.3-166.2$ & $<0.0001$ \\
\hline Thigh circumference (cm) & $49.2 \pm 5.4$ & $48.9 \pm 5.4$ & $16.0-105.0$ & $49.8 \pm 5.3$ & $24.6-99.2$ & $<0.0001$ \\
\hline Waist-to-hip ratio & $0.89 \pm 0.11$ & $0.88 \pm 0.12$ & $0.31-3.00$ & $0.91 \pm 0.09$ & $0.39-2.39$ & $<0.0001$ \\
\hline Neck-to-hip ratio & $0.37 \pm 0.05$ & $0.36 \pm 0.05$ & $0.17-1.30$ & $0.39 \pm 0.04$ & $0.24-1.24$ & $<0.0001$ \\
\hline Waist-to-thigh ratio & $1.71 \pm 0.22$ & $1.69 \pm 0.23$ & $0.58-4.91$ & $1.75 \pm 0.21$ & $0.69-3.77$ & $<0.0001$ \\
\hline Neck-to-thigh ratio & $0.71 \pm 0.09$ & $0.68 \pm 0.09$ & $0.32-2.67$ & $0.75 \pm 0.09$ & $0.40-2.14$ & $<0.0001$ \\
\hline Waist-to-neck ratio & $2.43 \pm 0.24$ & $2.48 \pm 0.25$ & $0.40-4.97$ & $2.35 \pm 0.23$ & $1.00-4.08$ & $<0.0001$ \\
\hline Hip-to-thigh ratio & $1.92 \pm 0.21$ & $1.92 \pm 0.22$ & $0.54-6.03$ & $1.92 \pm 0.20$ & $0.51-4.16$ & 0.82 \\
\hline \multicolumn{7}{|l|}{ Cardiometabolic disorders } \\
\hline Insulin resistance, n (\%) & $1814(29.1)$ & $1246(31.5)$ & / & $568(24.9)$ & / & $<0.0001$ \\
\hline Metabolic syndrome, n (\%) & $2199(35.2)$ & $1614(40.8)$ & / & $585(26.7)$ & / & $<0.0001$ \\
\hline $\begin{array}{l}\text { Elevated fasting blood glucose, } \\
\text { including diabetes, } \mathrm{n}(\%)\end{array}$ & $2423(38.8)$ & $1467(37.0)$ & / & $956(42.0)$ & / & 0.0001 \\
\hline High blood pressure, n (\%) & $4424(70.9)$ & 2795 (70.6) & / & $1629(71.5)$ & / & 0.44 \\
\hline High triglyceride, n (\%) & $2580(41.4)$ & $1723(43.5)$ & / & $857(37.6)$ & / & $<0.0001$ \\
\hline Low HDL cholesterol, n (\%) & $2102(33.7)$ & $1595(40.3)$ & / & $507(22.3)$ & / & $<0.0001$ \\
\hline
\end{tabular}

Data are presented as means \pm standard deviation (SD), or medians (inter-quartile ranges) for skewed variables, or numbers (proportions) for categorical variables. $P$ values were calculated from one-way analysis of variance (ANOVA) for continuous variables and chi-square test for categorical variables. Insulin resistance was defined as HOMA-IR $\geq 2.61$, which is the cut-off point for the highest quartile of the total participants

MET, metabolic equivalent task; HOMA-IR indicates homeostasis model assessment of insulin resistance; LDL cholesterol, low-density lipoprotein cholesterol; HDL cholesterol, high-density lipoprotein cholesterol 


\section{Distribution of body shapes}

The 8 distinct body shapes and their proportions by sex were shown in Fig. 1. 29.6\% of women and 28.0\% of men were assigned to the high WHR-high WTR-high WNR shape, $28.2 \%$ of women and $27.1 \%$ of men were the low WHR-low WTR-low WNR shape. Few individuals were assigned to the low WHR-high WTR-high WNR shape (women vs. men: $4.7 \%$ vs. $5.5 \%$ ) or the high WHR-low WTR-low WNR shape (women vs. men: $5.9 \%$ vs. $6.8 \%$ ). The proportions of other body shapes varied from 7.3 to $9.1 \%$.

\section{Body shape in relation to insulin resistance and cardiometabolic disorders}

The prevalence of insulin resistance and metabolic syndrome according to the 8 body shapes by sex were shown in Fig. 2. In women, as compared to low WHR-low WTRlow WNR shape, all body shapes were positively associated with insulin resistance. These associations were strongest for the high WHR-high WTR-low WNR shape (OR [95\% CI] was 3.71 [2.79-4.93], $P<0.0001$, Table 2). In men, as compared to low WHR-low WTR-low WNR shape, low WHR-high WTR-high WNR shape and the higher WHR related shapes were positively associated with insulin resistance. These associations were strongest for the high WHR-high WTR-low WNR shape (OR [95\% CI] was 3.00 [1.98-4.55], $P<0.0001$, Table 2).

The stability of body shapes was validated by the associations of body shapes with several major cardiometabolic disorders. In women, all body shapes were positively associated with metabolic syndrome and its components (elevated fasting blood glucose, high blood pressure, high triglycerides and low HDL cholesterol). These associations were strongest for the high WHRhigh WTR-low WNR shape (Table 2). In men, low WHRhigh WTR-high WNR shape and the higher WHR related shapes were associated with the higher risk of metabolic syndrome and its components. These associations were strongest for the high WHR-high WTR-low WNR shape (Table 2).

On consideration that WHR was the dominant determination indicator of insulin resistance and related cardiometabolic disorders. We further stratified the analysis by the WHR high- and low- levels (Table 3). In women,
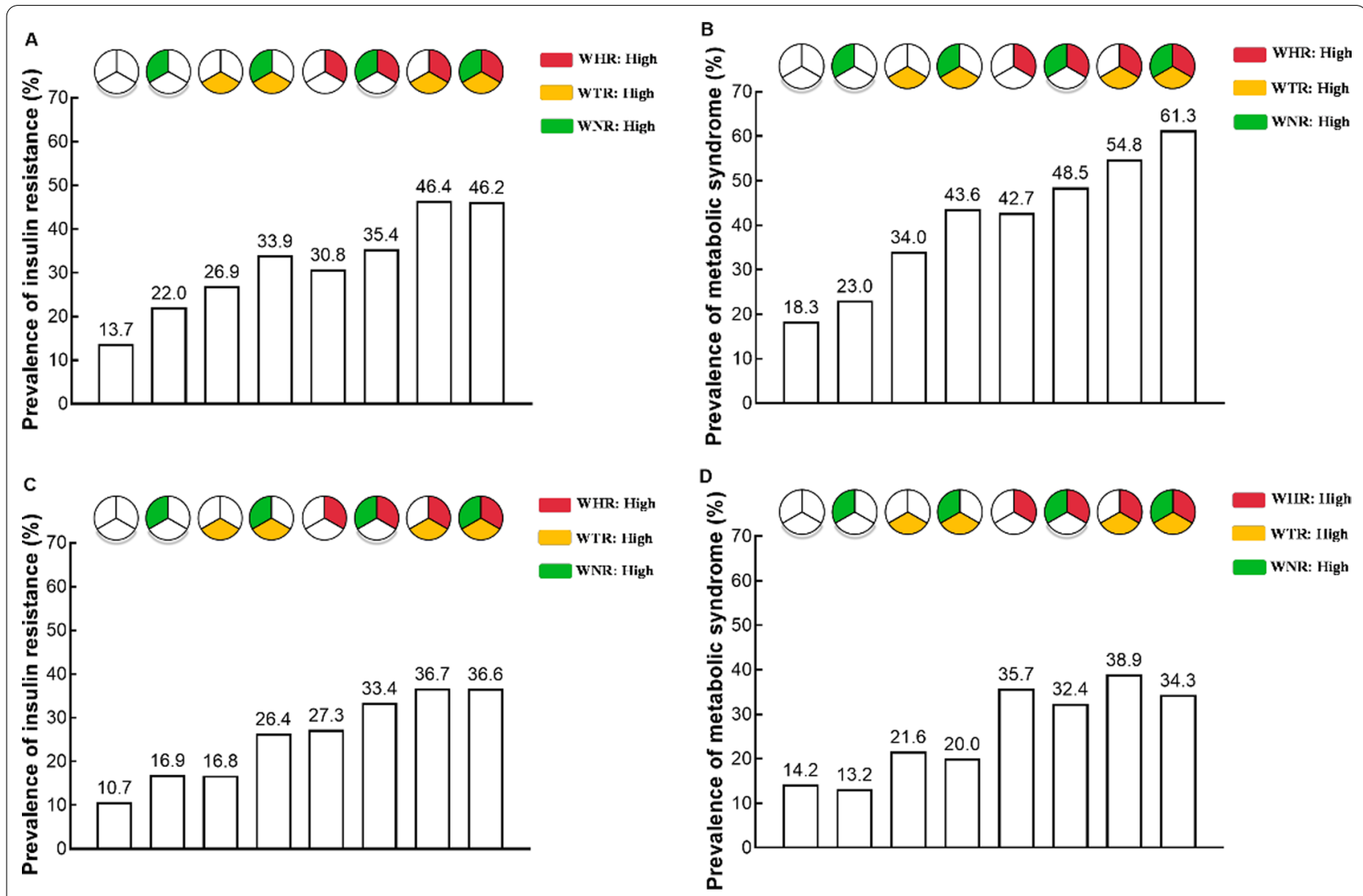

Fig. 2 The prevalence of insulin resistance and metabolic syndrome according to 8 body shapes by sex. A The prevalence of insulin resistance in women. B The prevalence of metabolic syndrome in women. CThe prevalence of insulin resistance in men. D The prevalence of metabolic syndrome in men. WHR, waist-to-hip ratio; WTR, waist-to-thigh ratio; WNR, waist-to-neck ratio 
Table 2 Association of body shape with risk of cardiovascular diseases in sex-specific samples

\begin{tabular}{|c|c|c|c|c|c|c|}
\hline Body shape & Insulin resistance & $\begin{array}{l}\text { Metabolic } \\
\text { syndrome }\end{array}$ & $\begin{array}{l}\text { Elevated fasting } \\
\text { blood glucose, } \\
\text { including diabetes }\end{array}$ & $\begin{array}{l}\text { High blood } \\
\text { pressure }\end{array}$ & High triglycerides & $\begin{array}{l}\text { Low HDL } \\
\text { cholesterol }\end{array}$ \\
\hline \multicolumn{7}{|l|}{ Women } \\
\hline $\begin{array}{l}\text { Low WHR-low WTR- } \\
\text { low WNR }\end{array}$ & Ref & Ref & Ref & Ref & Ref & Ref \\
\hline $\begin{array}{l}\text { Low WHR-low WTR- } \\
\text { high WNR }\end{array}$ & $1.49(0.89-1.75)$ & $0.96(0.70-1.33)$ & $0.99(0.74-1.34)$ & $0.85(0.65-1.12)$ & $1.41(1.08-1.85)^{*}$ & $1.09(0.83-1.44)$ \\
\hline $\begin{array}{l}\text { Low WHR-high WTR- } \\
\text { low WNR }\end{array}$ & $2.09(1.51-2.88)^{*}$ & $1.97(1.47-2.65)^{*}$ & $1.77(1.34-2.33)^{*}$ & $1.59(1.18-2.14)^{*}$ & $1.57(1.20-2.05)^{*}$ & $1.79(1.37-2.33)^{*}$ \\
\hline $\begin{array}{l}\text { Low WHR-high WTR- } \\
\text { high WNR }\end{array}$ & $2.09(1.44-3.02)^{*}$ & $2.17(1.53-3.06)^{*}$ & $2.32(1.67-3.23)^{*}$ & $1.27(0.88-1.84)$ & $1.90(1.38-2.63)^{*}$ & $1.15(0.82-1.61)$ \\
\hline $\begin{array}{l}\text { High WHR-low WTR- } \\
\text { low WNR }\end{array}$ & $2.02(1.44-2.84)^{*}$ & $2.57(1.89-3.51)^{*}$ & $1.31(0.96-1.80)$ & $1.54(1.13-2.12)^{*}$ & $2.26(1.69-3.01)^{*}$ & $2.14(1.60-2.86)^{*}$ \\
\hline $\begin{array}{l}\text { High WHR-low WTR- } \\
\text { high WNR }\end{array}$ & $1.92(1.41-2.61)^{*}$ & $2.59(1.94-3.45)^{*}$ & $1.37(1.03-1.83)^{*}$ & $1.78(1.31-2.40)^{*}$ & $2.25(1.73-2.93)^{*}$ & $1.77(1.36-2.31)^{*}$ \\
\hline $\begin{array}{l}\text { High WHR-high } \\
\text { WTR-low WNR }\end{array}$ & $3.71(2.79-4.93)^{*}$ & $3.57(2.72-4.69)^{*}$ & $2.36(1.82-3.06)^{*}$ & $2.23(1.63-3.06)^{*}$ & $3.15(2.44-4.06)^{*}$ & $2.13(1.65-2.74)^{*}$ \\
\hline $\begin{array}{l}\text { High WHR-high } \\
\text { WTR-high WNR }\end{array}$ & $2.84(2.25-3.58)^{*}$ & $3.81(3.08-4.71)^{*}$ & $1.94(1.59-2.38)^{*}$ & $1.85(1.49-2.30)^{*}$ & $2.33(1.93-2.82)^{*}$ & $1.66(1.37-2.01)^{*}$ \\
\hline \multicolumn{7}{|l|}{ Men } \\
\hline $\begin{array}{l}\text { Low WHR-low WTR- } \\
\text { low WNR }\end{array}$ & Ref & Ref & Ref & Ref & Ref & Ref \\
\hline $\begin{array}{l}\text { Low WHR-low WTR- } \\
\text { high WNR }\end{array}$ & $1.23(0.76-1.98)$ & $0.67(0.41-1.10)$ & $0.73(0.51-1.05)$ & $0.78(0.55-1.11)$ & $1.09(0.75-1.59)$ & $0.97(0.62-1.53)$ \\
\hline $\begin{array}{l}\text { Low WHR-high WTR- } \\
\text { low WNR }\end{array}$ & $1.49(0.91-2.44)$ & $1.50(0.96-2.34)$ & $1.30(0.91-1.85)$ & $1.08(0.74-1.58)$ & $1.85(1.27-2.69)^{*}$ & $1.30(0.83-2.04)$ \\
\hline $\begin{array}{l}\text { Low WHR-high WTR- } \\
\text { high WNR }\end{array}$ & $2.41(1.48-3.93)^{*}$ & $1.20(0.73-2.00)$ & $1.25(0.84-1.87)$ & $1.00(0.65-1.54)$ & $1.28(0.82-1.98)$ & $1.04(0.61-1.77)$ \\
\hline $\begin{array}{l}\text { High WHR-low WTR- } \\
\text { low WNR }\end{array}$ & $2.11(1.38-3.32)^{*}$ & $2.32(1.54-3.52)^{*}$ & $1.83(1.27-2.64)^{*}$ & $1.29(0.86-1.93)$ & $2.48(1.70-3.63)^{*}$ & $2.02(1.33-3.06)^{*}$ \\
\hline $\begin{array}{l}\text { High WHR-low WTR- } \\
\text { high WNR }\end{array}$ & $2.11(1.39-3.20)^{*}$ & $1.61(1.09-2.39)^{*}$ & $1.37(0.98-1.92)$ & $1.01(0.70-1.47)$ & $2.43(1.71-3.46)^{*}$ & $2.11(1.44-3.11)^{*}$ \\
\hline $\begin{array}{l}\text { High WHR-high } \\
\text { WTR-low WNR }\end{array}$ & $3.00(1.98-4.55)^{*}$ & $2.48(1.67-3.67)^{*}$ & $1.81(1.28-2.57)^{*}$ & $2.01(1.30-3.11)^{*}$ & $2.48(1.73-3.57)^{*}$ & $2.00(1.34-2.99)^{*}$ \\
\hline $\begin{array}{l}\text { High WHR-high } \\
\text { WTR-high WNR }\end{array}$ & $2.62(1.87-3.66)^{*}$ & $1.80(1.32-2.46)^{*}$ & $1.51(1.17-1.94)^{*}$ & $1.70(1.26-2.28)^{*}$ & $1.89(1.44-2.48)^{*}$ & $1.43(1.04-1.96)^{*}$ \\
\hline
\end{tabular}

Data were presented as odds ratio (OR) and 95\% confidence interval (CI). $P$ values were calculated from multivariable logistic regression analysis. Adjusted age (years), body mass index $\left(\mathrm{kg} / \mathrm{m}^{2}\right)$, current smoking (yes or no), current drinking (yes or no), and physical activity (MET-h/wk). According to the medians, WHR, WTR, and WNR were divided into high and low levels. The corresponding median value for WHR is 0.87 in women and 0.91 for men, for WTR is 1.66 in women and 1.74 for men, and for WNR is 2.48 in women and 2.34 in men

*, $P<0.05$; HDL cholesterol, high-density lipoprotein cholesterol; WHR, waist-to-hip ratio; WTR, waist-to-thigh ratio; WNR, waist-to-neck ratio

both in the high and low-WHR groups, those with high WTR shapes were significantly associated high risk of insulin resistance and related cardiometabolic disorders. In men, nearly all body shapes were not associated with insulin resistance and the related cardiometabolic disorders when stratified by WHR level (all $P>0.05$, Table 3 ).

\section{Predictive values of anthropometric traits}

In women, for insulin resistance, the addition of WHR, WTR, and WNR to a model containing BMI resulted in a change in the $C$ statistic from 0.740 to 0.757 , an NRI of 0.350 , and an IDI 0.020 (all $P<0.001$ ).
For metabolic syndrome, the addition of WHR, WTR, and WNR to a model containing BMI resulted in a change in the $C$ statistic from 0.743 to 0.787 , an NRI of 0.368 , and an IDI 0.034 (all $P<0.001$ ). The combination of BMI, WHR, WTR, and WNR significantly increased $C$ statistic, NRI and IDI for predicting the risk of insulin resistance and metabolic syndrome. In men, the inclusion of WHR, WTR, and WNR to a model containing BMI for predicting insulin resistance and metabolic syndrome significantly increased NRI and IDI (all $P \leq 0.04$ ), except for $C$ statistic (Additional file 1: Table S1). 
Table 3 Stratified analysis for associations body shape with insulin resistance and cardiometabolic disorders by WHR

\begin{tabular}{|c|c|c|c|c|c|c|}
\hline Body shape & Insulin resistance & $\begin{array}{l}\text { Metabolic } \\
\text { syndrome }\end{array}$ & $\begin{array}{l}\text { Elevated fasting } \\
\text { blood glucose, } \\
\text { including diabetes }\end{array}$ & $\begin{array}{l}\text { High blood } \\
\text { pressure }\end{array}$ & High triglycerides & $\begin{array}{l}\text { Low HDL } \\
\text { cholesterol }\end{array}$ \\
\hline \multicolumn{7}{|l|}{ Women } \\
\hline $\begin{array}{l}\text { Low WHR-low WTR- } \\
\text { low WNR }\end{array}$ & Ref & Ref & Ref & Ref & Ref & Ref \\
\hline $\begin{array}{l}\text { Low WHR-low WTR- } \\
\text { high WNR }\end{array}$ & $1.49(0.89-1.75)$ & $0.96(0.70-1.33)$ & $0.99(0.74-1.34)$ & $0.85(0.65-1.12)$ & $1.41(1.08-1.85)^{*}$ & $1.09(0.83-1.44)$ \\
\hline $\begin{array}{l}\text { Low WHR-high WTR- } \\
\text { low WNR }\end{array}$ & $2.10(1.51-2.88)^{*}$ & $1.97(1.47-2.65)^{*}$ & $1.77(1.34-2.33)^{*}$ & $1.59(1.18-2.14)^{*}$ & $1.57(1.20-2.05)^{*}$ & $1.79(1.37-2.33)^{*}$ \\
\hline $\begin{array}{l}\text { Low WHR-high WTR- } \\
\text { high WNR }\end{array}$ & $2.09(1.44-3.02)^{*}$ & $2.17(1.53-3.06)^{*}$ & $2.32(1.67-3.23)^{*}$ & $1.27(0.88-1.84)$ & $1.90(1.38-2.63)^{*}$ & $1.15(0.82-1.61)$ \\
\hline $\begin{array}{l}\text { High WHR-low WTR- } \\
\text { low WNR }\end{array}$ & Ref & Ref & Ref & Ref & Ref & Ref \\
\hline $\begin{array}{l}\text { High WHR-low WTR- } \\
\text { high WNR }\end{array}$ & $0.95(0.65-1.39)$ & $1.01(0.71-1.44)$ & $1.05(0.72-1.51)$ & $1.15(0.78-1.70)$ & $1.00(0.71-1.41)$ & $0.83(0.59-1.17)$ \\
\hline $\begin{array}{l}\text { High WHR-high } \\
\text { WTR-low WNR }\end{array}$ & $1.84(1.28-2.64)^{*}$ & $1.39(0.98-1.96)$ & $1.79(1.26-2.55)^{*}$ & $1.45(0.97-2.16)$ & $1.40(1.00-1.95)$ & $0.99(0.71-1.39)$ \\
\hline $\begin{array}{l}\text { High WHR-high } \\
\text { WTR-high WNR }\end{array}$ & $1.41(1.02-1.93)^{*}$ & $1.48(1.10-2.00)^{*}$ & $1.49(1.09-2.01)^{*}$ & $1.20(0.86-1.67)$ & $1.03(0.78-1.38)$ & $0.76(0.58-1.03)$ \\
\hline \multicolumn{7}{|l|}{ Men } \\
\hline $\begin{array}{l}\text { Low WHR-low WTR- } \\
\text { low WNR }\end{array}$ & Ref & Ref & Ref & Ref & Ref & Ref \\
\hline $\begin{array}{l}\text { Low WHR-low WTR- } \\
\text { high WNR }\end{array}$ & $1.23(0.76-1.98)$ & $0.67(0.41-1.10)$ & $0.73(0.51-1.05)$ & $0.78(0.55-1.11)$ & $1.09(0.75-1.59)$ & $0.97(0.62-1.53)$ \\
\hline $\begin{array}{l}\text { Low WHR-high WTR- } \\
\text { low WNR }\end{array}$ & $1.49(0.91-2.44)$ & $1.50(0.96-2.34)$ & $1.30(0.91-1.85)$ & $1.08(0.74-1.58)$ & $1.85(1.27-2.69)^{*}$ & $1.30(0.83-2.04)$ \\
\hline $\begin{array}{l}\text { Low WHR-high WTR- } \\
\text { high WNR }\end{array}$ & $2.41(1.48-3.93)^{*}$ & $1.20(0.73-2.00)$ & $1.25(0.84-1.87)$ & $1.00(0.65-1.54)$ & $1.28(0.82-1.98)$ & $1.04(0.61-1.77)$ \\
\hline $\begin{array}{l}\text { High WHR-low WTR- } \\
\text { low WNR }\end{array}$ & Ref & Ref & Ref & Ref & Ref & Ref \\
\hline $\begin{array}{l}\text { High WHR-low WTR- } \\
\text { high WNR }\end{array}$ & $1.00(0.62-1.61)$ & $0.69(0.44-1.09)$ & $0.75(0.40-1.14)$ & $0.79(0.49-1.27)$ & $0.98(0.64-1.51)$ & $1.05(0.66-1.65)$ \\
\hline $\begin{array}{l}\text { High WHR-high } \\
\text { WTR-low WNR }\end{array}$ & $1.42(0.88-2.30)$ & $1.07(0.68-1.68)$ & $0.99(0.64-1.53)$ & $1.56(0.92-2.66)$ & $1.00(0.64-1.56)$ & $0.99(0.62-1.59)$ \\
\hline $\begin{array}{l}\text { High WHR-high } \\
\text { WTR-high WNR }\end{array}$ & $1.24(0.83-1.87)$ & $0.77(0.53-1.14)$ & $0.82(0.57-1.18)$ & $1.32(0.86-2.01)$ & $0.76(0.53-1.10)$ & $0.71(0.47-1.05)$ \\
\hline
\end{tabular}

Data were presented as odds ratio (OR) and $95 \%$ confidence interval (CI). $P$ values were calculated from multivariable logistic regression analysis. Adjusted age (years), body mass index $\left(\mathrm{kg} / \mathrm{m}^{2}\right)$, current smoking (yes or no), current drinking (yes or no), and physical activity (MET-h/wk). According to the medians, WHR, WTR, and WNR were divided into high and low levels. The corresponding median value for WHR is 0.87 in women and 0.91 for men, for WTR is 1.66 in women and 1.74 for men, and for WNR is 2.48 in women and 2.34 in men

*, $P<0.05$; HDL cholesterol, high-density lipoprotein cholesterol; WHR, waist-to-hip ratio; WTR, waist-to-thigh ratio; WNR, waist-to-neck ratio

We further compared the predictive value between "BMI + WHR + WNR + WTR" and "BMI + WHR" in evaluating insulin resistance and metabolic syndrome in total sample, $\mathrm{BMI}<24 \mathrm{~kg} / \mathrm{m}^{2}$ group, and BMI $\geq 24 \mathrm{~kg} / \mathrm{m}^{2}$ group (Additional file 1: Table S3). We found "BMI + WHR + WNR + WTR" was significantly superior to "BMI + WHR" in predicting the risk of insulin resistance and metabolic syndrome in women with BMI less than $24 \mathrm{~kg} / \mathrm{m}^{2}$, the $\Delta C$ statistic was 0.027 (0.012-0.042), 0.030 (0.010-0.049), respectively (all $P<0.003)$.

\section{Sensitivity analysis}

We randomly selected $80 \%(\mathrm{n}=4492)$ individuals as training sample and performed logistic regression analyses of body shapes with insulin resistance and metabolic syndrome. The predictive value of BMI and $\mathrm{BMI}+\mathrm{WHR}+\mathrm{WTR}+\mathrm{WNR}$ from the training sample were consistent with our main analysis. Additionally, in the training sample, we compared the sensitivity and specificity of BMI + WHR + WTR + WNR with BMI for diagnosing insulin resistance and metabolic syndrome. 
For insulin resistance, the inclusion of WHR, WTR, and WNR to a model containing BMI resulted in sensitivity changed from $60.1 \%$ to $64.7 \%$ in women, $(63.1 \%$ to $64.5 \%$ in men); specificity changed from $75.8 \%$ to $72.3 \%$ in women ( $71.3 \%$ to $70.5 \%$ in men). For metabolic syndrome, the inclusion of WHR, WTR, and WNR to a model containing BMI resulted in sensitivity changed from $68.7 \%$ to $73.3 \%$ in women $(57.8 \%$ to $60.4 \%$ in men), specificity changed from 66.8 to $63.4 \%$ in women $(72.1 \%$ to $70.1 \%$ in men) (Additional file 1: Fig. S2).

We further compared the predictive value of multiple anthropometric traits in insulin resistance and metabolic syndrome in the test sample (the remaining $20 \%, n=778)$. In women, for insulin resistance, the addition of weighted $\mathrm{BMI}+\mathrm{WHR}+\mathrm{WTR}+\mathrm{WNR}$ to a model containing BMI resulted in $\mathrm{C}$ statistic changed from 0.713 to 0.729 , sensitivity changed from $75.8 \%$ to $82.3 \%$, specificity changed from $57.6 \%$ to $51.7 \%$, an NRI of 0.579 , and an IDI of 0.025 (all $P<0.002$, Additional file 1: Fig. S2). For metabolic syndrome, when weighted $\mathrm{BMI}+\mathrm{WHR}+\mathrm{WTR}+\mathrm{WNR}$ was added to a model with BMI, C-statistic changed from 0.724 to 0.760 , sensitivity changed from $55.7 \%$ to $75.5 \%$, specificity change from $78.0 \%$ to $66.0 \%$, an NRI of 0.616 , and an IDI of 0.054 (all $P<0.001$, Additional file 1: Fig. S2). In men, the IDI significantly increased for insulin resistance and metabolic syndrome (all $P<0.05$, Additional file 1: Fig. S2). These results indicated that the combination of multiple anthropometric traits can enhance the sensitivity for diagnosing cardiometabolic disorders.

\section{Discussion}

In this cross-sectional study of 6240 community-dwelling Chinese adults, we defined 8 distinct body shapes based on sex-specific combinations of WHR, WTR and WNR, which differed in participants' distribution and risk of insulin resistance and cardiometabolic disorders. In women, as compared to the low WHR-low WTR-low WNR shape, all the body shapes were significantly associated with a higher risk of IR and related cardiometabolic disorders. In men, as compared to low WHR-low WTR-low WNR shape, low WHR-high WTR-high WNR shape and the higher WHR related shapes were positively associated with insulin resistance and related cardiometabolic disorders. The risk of cardiometabolic disorders identified by multiple anthropometric traits independent of BMI and WHR, suggesting that the combination of multiple novel anthropometrics would benefit to identify additional cardiometabolic risk.

Emerging studies had illustrated that anthropometric traits were well-established predictors for insulin resistance, metabolic syndrome, type 2 diabetes and cardiovascular disease [7, 12, 14, 24]. For example, neck circumference and thinner thigh circumference were associated with an increased risk of peripheral arterial disease, cardiometabolic diseases and premature death $[25,26]$. However, these studies only focused on solitary index rather than whole-body shape measured by multiple anthropometric traits, which couldn't discriminate the difference of diseases susceptibility related to body shape diversity. Therefore, our study takes 3 ratios, WHR, WTR, and WNR, simultaneously into account to define 8 distinct body shapes, and evaluate their associations with risk of insulin resistance and cardiometabolic disorders. These body shapes showed distinct cardiometabolic risk in women.

In the current study, we compared the diagnostic performance of these anthropometric traits with routine clinical parameters (BMI). The results showed that the C-statistic improved slightly, the sensitivity improved, and the specificity decreased with the added anthropometric measures. C-statistic was widely used for the evaluation of risk prediction models. But it was insensitive as it often fails to detect improvements in the prediction that result from adding clinically relevant risk factors [27]. For example, adding novel indicators might increase the risk differences without improving discriminative ability when the $\mathrm{C}$-statistic of the clinical prediction model is already high [27]. Although the C-statistic improved slightly in the present study, a clinically meaningful improvement in the NRI, IDI, and sensitivity was detected. In addition, in our study, the combination of $\mathrm{BMI}+\mathrm{WHR}+\mathrm{WTR}+\mathrm{WNR}$ improved sensitivity without significantly reducing specificity. These results indicated that the combination of multiple anthropometric measures might increase the power to predict cardiometabolic risk, and was appropriate to large-scale epidemiological survey to identify more potential high-risk individuals. Our findings provide information for the development of better initial screening tools for cardiometabolic risk.

Mechanically, muscle mass loss of extremity circumference could influence insulin sensitivity, fat oxidation, and glucose metabolism and promote metabolic disorders $[28,29]$. Neck fat accumulations could increase secretion of proinflammatory cytokine, elevated free fatty acid production, exacerbated systematic inflammation and impact glucose and lipid metabolism [30, 31]. These factors together exacerbated the risk of insulin resistance and related cardiometabolic disorders.

WHR was the dominant determination indicator of insulin resistance and related cardiometabolic disorders. In addition to WHR level, the WTR was the secondary determination indicator of insulin resistance and related cardiometabolic disorders in women. These results indicated that WTR may play a complementary role to WHR 
in predicting risk for cardiometabolic disorders. However, no significant associations were detected in men when stratified by the WHR level. Plausible explanations were biological differences between men and women, such as sex hormones metabolism, immune system responses, redistribution of body fat, muscle capacity and physical function [32, 33].

To the best of our knowledge, it is the first study to define refined body shapes by using multiple anthropometric traits simultaneously. The WHR, WTR, and WNR can represent the upper-, central-and lower- body fat distribution, and their measurements are convenient, simple, low cost, and harmless, which may benefit to refine define body shape and suit for large-scale population-based study. Besides, the well-defined community setting, fair sized sample volume, and desirable population homogeneity were a great foundation for current analysis. Several limitations should be acknowledged. Firstly, we could not establish a causal relationship between body shapes and the cardiometabolic risk. Due to the cross-sectional nature of this study, we only assessed the predictive ability of these anthropometric traits on the prevalence of cardiometabolic disorders. We could not obtain a "true predictive relationship" between body shape and future metabolic risk. The prospective follow-up studies are warranted to further verify the results in the current study. Secondly, the onset of cardiometabolic diseases is insidious, mostly occurring aged 40 years or above. Body composition changed with aging. Our study was conducted in middle and elderly adults, and it should be cautious to interpret the results to the youngers. Thirdly, adopting multiple anthropometric traits to define a comprehensive body shape is more complicated than BMI and WHR in clinical practice. However, a recent study indicated that the combination of multiple indicators would provide a powerful tool to predict metabolic disorders [34]. Our study presented a whole picture of the body shape defined by multiple anthropometric parameters other than BMI, which could capture additional cardiometabolic risk and may provide a useful value in disease screening. Fourthly, the cut-off value of WHR, WTR, and WNR in distinguishing cardiometabolic risk was just based on a statistical significance, it is far to be used as a diagnostic standard. The WHR, WTR, and WNR cut-off values should be further verified in larger prospective cohorts in different age or ethnicity groups.

\section{Conclusions}

In conclusion, we defined 8 distinct body shapes by taking WHR, WTR, and WNR, simultaneously into account in a Chinese community-based cohort. The combination of WHR, WTR, and WNR was significantly associated with a higher risk of insulin resistance and cardiometabolic disorders independent of BMI or WHR. The defined body shapes do provide additional cardiometabolic risk assessment over BMI and WHR. Our findings provided a useful, simple, and available method to discriminate parts of individual variation in the cardiometabolic risk.

\section{Abbreviations}

BMI: Body mass index; IR: Insulin resistance; WHR: Waist-to-hip ratio; WTR: Waist-to-thigh ratio; WNR: Waist-to-neck ratio; OGTT: Oral glucose tolerance tests; HDL: Cholesterol, high-density lipoprotein cholesterol; LDL: Cholesterol, low-density lipoprotein cholesterol; HOMA-IR: Homeostasis model assessment of insulin resistance; NRI: Net reclassification improvement; IDI: Integrated discrimination improvement.

\section{Supplementary Information}

The online version contains supplementary material available at https://doi. org/10.1186/s12986-021-00629-1.

Additional file 1: Figure S1. Feature selection. A: Automated feature selection. It was performed by using the information gain attribute ranking method on the open-source Waikato Environment for Knowledge Analysis platform. Information gain ranking was used to evaluate the worth of each variable (usually the clinical indicator) by measuring the entropy gain to the outcome. The greater the information gain a clinical indicator has, the more important the indicator is in the classification process. The model was built with logistic regression analysis based on the data set. The regression coefficient of each significant variable was regarded as the contribution level. B: Entropy gain for each indicator. It illuminates the information gain and ranks the attributes of each variable from the top to the bottom. Three indicators (WHR, WNR and WTR) were chosen to construct body shapes. Abbreviation: WHR, waist-to-hip ratio; WTR, waist-to-thigh ratio; WNR, waist-to-neck ratio; NTR, neck-to-thigh ratio; NHR, neck-to-hip ratio; THR, thigh-to-hip ratio. Figure S2. Predictive values of anthropometric traits in training sample and test sample. The predictive value of $\mathrm{BMI}$ and $\mathrm{BMI}+\mathrm{WHR}+\mathrm{WTR}+\mathrm{WNR}$ for diagnosing insulin resistance and metabolic syndrome in training sample $(A, B)$. The predictive value of $\mathrm{BMI}$ and $\mathrm{BMI}+\mathrm{WHR}+\mathrm{WTR}+\mathrm{WNR}$ for diagnosing insulin resistance and metabolic syndrome in testing sample (C, D). Data are C statistic, $\triangle C$ statistic, IDI and NRI, 95\% confidence intervals, sensitivity, specificity. $P$ values were from logistic analysis. Abbreviation: NRI, net reclassification improvement; IDI, integrated dis-crimination improvement; BMI, body mass index; WHR, waist-to-hip circumference ratio; WTR, waist-to-thigh circumference ratio; WNR, waist-to-neck circumference ratio. * indicated $P$ value $<0.05$. Table S1. Predictive values of anthropometric traits in total sample. Table S2. Association of body shape with risk of cardiovascular diseases in training sample. Table S3. Predictive values of anthropometric traits in stratification analysis.

\section{Acknowledgements}

We thank all the study participants for their participation and contribution. The present study would not have been possible without the participation of the participants

\section{Authors' contributions}

$Y Z, Y H, M X$, and $Z Z$ conceived and designed the study. $Y Z$ and $Y H$ did the statistical analysis. $Y H$ and $Z Z$ did the model building. $Y H$ and $M X$ drafted the manuscript. ZZ, YB, and MX supervised the study. HD, ML, HL, SW, YX, JL, YC, $W W$, and ZZ contributed to acquisition, analysis, or interpretation of data. All authors revised the report and approved the final version before submission. MX and ZZ are the guarantors of this work and, as such, had full access to all the data in the study and take responsibility for the integrity of the data and 
the accuracy of the data analysis. All authors read and approved the final manuscript.

\section{Funding}

This work was supported by grants from the National Natural Science Foundation of China $(82070880,81941017,81770842,81970728,81970706$, and 81870560), the National Key R\&D Program of China (2018YFC1311705, 2016YFC1305600, and 2016YFC1304904), the Clinical Research Plan of SHDC (SHDC2020CR1001A and SHDC2020CR3069B), and the Shanghai Municipal Education Commission-Gaofeng Clinical Medicine Grant Support (20161307 and 20152508 Round 2). M. X., J. L., M. L., T.W., Y. X., Z. Z., Y. B., W. W., and G. N. are members of innovative research team of high-level local universities in Shanghai.

\section{Availability of data and materials}

All data generated or analyzed during this study are included in this published article (and its Additional information files).

\section{Declarations}

\section{Ethics approval and consent to participate}

The study protocol was approved by the Institutional Review Board of Ruijin Hospital, Shanghai Jiao Tong University School of Medicine and procedures were in accordance with the ethical standards of the responsible committee on human experimentation and with the Helsinki declaration of 1975, as revised in 1983. Written informed consent was obtained from each study participant.

\section{Consent for publication}

Not applicable.

\section{Competing interest}

The authors declare that they have no competing interests.

\section{Author details}

${ }^{1}$ Department of Endocrine and Metabolic Diseases, Shanghai Institute of Endocrine and Metabolic Diseases, Ruijin Hospital, Shanghai Jiao Tong University School of Medicine, 197 Ruijin 2nd Road, 200025 Shanghai, China.

${ }^{2}$ Shanghai National Clinical Research Center for Metabolic Diseases, Key Laboratory for Endocrine and Metabolic Diseases of the National Health Commission of the PR China, Shanghai National Center for Translational Medicine, Ruijin Hospital, Shanghai Jiao Tong University School of Medicine, Shanghai, China.

Received: 9 June 2021 Accepted: 19 November 2021

Published online: 07 December 2021

\section{References}

1. Kumanyika S, Dietz WH. Solving population-wide obesity-progress and future prospects. N Engl J Med. 2020;383:2197-200.

2. Dai H, Alsalhe TA, Chalghaf N, Ricco M, Bragazzi NL, Wu J. The global burden of disease attributable to high body mass index in 195 countries and territories, 1990-2017: an analysis of the Global Burden of Disease Study. PLoS Med. 2020;17:e1003198.

3. Li X, Wu C, Lu J, Chen B, Li Y, Yang Y, et al. Cardiovascular risk factors in China: a nationwide population-based cohort study. Lancet Public Health. 2020;5:e672-81.

4. Stefan N. Causes, consequences, and treatment of metabolically unhealthy fat distribution. Lancet Diabetes Endocrinol. 2020;8:616-27.

5. Norris T, Cole TJ, Bann D, Hamer M, Hardy R, Li L, et al. Duration of obesity exposure between ages 10 and 40 years and its relationship with cardiometabolic disease risk factors: a cohort study. PLoS Med. 2020;17:e1003387.

6. Soares ALG, Banda L, Amberbir A, Jaffar S, Musicha C, Price AJ, et al. A comparison of the associations between adiposity and lipids in Malawi and the United Kingdom. BMC Med. 2020;18:181.
7. Emdin CA, Khera AV, Natarajan P, Klarin D, Zekavat SM, Hsiao AJ, et al. Genetic association of waist-to-hip ratio with cardiometabolic traits, type 2 diabetes, and coronary heart disease. JAMA. 2017;317:626-34

8. Trepanowski JF, Kroeger CM, Barnosky A, Klempel MC, Bhutani $\mathrm{S}$, Hoddy $\mathrm{KK}$, et al. Effect of alternate-day fasting on weight loss, weight maintenance, and cardioprotection among metabolically healthy obese adults: a randomized clinical trial. JAMA Intern Med. 2017;177:930-8.

9. Teufel F, Seiglie JA, Geldsetzer P, Theilmann M, Marcus ME, Ebert C, et al. Body-mass index and diabetes risk in 57 low-income and middleincome countries: a cross-sectional study of nationally representative, individual-level data in 685616 adults. Lancet. 2021;398:238-48.

10. Hruby A, Hu FB. The epidemiology of obesity: a big picture. Pharmacoeconomics. 2015:33:673-89.

11. Lavie CJ, De Schutter A, Patel DA, Romero-Corral A, Artham SM, Milani RV. Body composition and survival in stable coronary heart disease: impact of lean mass index and body fat in the "obesity paradox." J Am Coll Cardiol. 2012:60:1374-80.

12. Kamiya K, Masuda T, Matsue Y, Inomata T, Hamazaki N, Matsuzawa R, et al. Complementary role of arm circumference to body mass index in risk stratification in heart failure. JACC Heart Fail. 2016;4:265-73.

13. Gastelurrutia P, Lupon J, de Antonio M, Zamora E, Domingo M, Urrutia A, et al. Body mass index, body fat, and nutritional status of patients with heart failure: the PLICA study. Clin Nutr. 2015;34:1233-8.

14. Wu CJ, Kao TW, Chang YW, Peng TC, Wu LW, Yang HF, et al. Does the additional component of calf circumference refine metabolic syndrome in correlating with cardiovascular risk? J Clin Endocrinol Metab. 2018;103:1151-60

15. Jayedi A, Soltani S, Zargar MS, Khan TA, Shab-Bidar S. Central fatness and risk of all cause mortality: systematic review and dose-response meta-analysis of 72 prospective cohort studies. BMJ. 2020;370:m3324

16. Lee JJ, Pedley A, Therkelsen KE, Hoffmann U, Massaro JM, Levy D, et al. Upper body subcutaneous fat is associated with cardiometabolic risk factors. Am J Med. 2017;130:958-66.

17. Huang Y, Bi Y, Xu M, Ma Z, Xu Y, Wang T, et al. Nonalcoholic fatty liver disease is associated with atherosclerosis in middle-aged and elderly Chinese. Arterioscler Thromb Vasc Biol. 2012;32:2321-6.

18. Lin L, Zhang J, Jiang L, Du R, Hu C, Lu J, et al. Transition of metabolic phenotypes and risk of subclinical atherosclerosis according to BMI: a prospective study. Diabetologia. 2020;63:1312-23.

19. Craig CL, Marshall AL, Sjöström M, Bauman AE, Booth ML, Ainsworth $B E$, et al. International physical activity questionnaire: 12-country reliability and validity. Med Sci Sports Exerc. 2003;35:1381-95.

20. Heitmann BL, Frederiksen P. Thigh circumference and risk of heart disease and premature death: prospective cohort study. BMJ. 2009;339:b3292.

21. Ben-Noun L, Sohar E, Laor A. Neck circumference as a simple screening measure for identifying overweight and obese patients. Obes Res. 2001:9:470-7.

22. Grundy SM, Cleeman JI, Daniels SR, Donato KA, Eckel RH, Franklin BA, et al. Diagnosis and management of the metabolic syndrome: an American Heart Association/National Heart, Lung, and Blood Institute Scientific Statement. Circulation. 2005:112:2735-52.

23. Ling SY, Zhao ZY, Tao B, Zhao HY, Su TW, Jiang YR, et al. Pituitary stalk thickening in a large cohort: toward more accurate predictors of pituitary dysfunction and etiology. Endocr Pract. 2019;25:534-44.

24. Sumner $A E$, Bagheri $M H$. Identifying the waist circumference of risk in people of African descent. Nat Rev Endocrinol. 2020;16:1-3.

25. Min JY, Cho JS, Lee KJ, Park JB, Min KB. Thigh circumference and low ankle brachial index in US adults: results from the National Health and Nutrition Examination Survey 1999-2004. Int J Cardiol. 2013;163:40-5.

26. Preis SR, Pencina MJ, D'Agostino RB Sr, Meigs JB, Vasan RS, Fox CS. Neck circumference and the development of cardiovascular disease risk factors in the Framingham Heart Study. Diabetes Care. 2013;36:e3.

27. Martens FK, Tonk ECM, Janssens A. Evaluation of polygenic risk models using multiple performance measures: a critical assessment of discordant results. Genet Med. 2019;21:391-7.

28. Pedersen BK, Febbraio MA. Muscles, exercise and obesity: skeletal muscle as a secretory organ. Nat Rev Endocrinol. 2012;8:457-65.

29. Winkler TW, Justice AE, Graff M, Barata L, Feitosa MF, Chu S, et al. The influence of age and sex on genetic associations with adult body size 
and shape: a large-scale genome-wide interaction study. PLoS Genet. 2015;11:e1005378.

30. Luo Y, Ma X, Shen Y, Xu Y, Xiong Q, Zhang X, et al. Neck circumference as an effective measure for identifying cardio-metabolic syndrome: a comparison with waist circumference. Endocrine. 2017;55:822-30.

31. Mason C, Craig CL, Katzmarzyk PT. Influence of central and extremity circumferences on all-cause mortality in men and women. Obesity (Silver Spring). 2008;16:2690-5.

32. Wu LW, Lin YY, Kao TW, Lin CM, Wang CC, Wang GC, et al. Mid-arm circumference and all-cause, cardiovascular, and cancer mortality among obese and non-obese US adults: the national health and nutrition examination survey III. Sci Rep. 2017;7:2302.

33. Wells JC, Treleaven P, Cole TJ. BMI compared with 3-dimensional body shape: the UK National Sizing Survey. Am J Clin Nutr. 2007:85:419-25.

34. Shi J, Bao G, Hong J, Wang S, Chen Y, Zhao S, et al. Deciphering CT texture features of human visceral fat to evaluate metabolic disorders and surgery-induced weight loss effects. EBioMedicine. 2021;69:103471.

\section{Publisher's Note}

Springer Nature remains neutral with regard to jurisdictional claims in published maps and institutional affiliations.

- fast, convenient online submission

- thorough peer review by experienced researchers in your field

- rapid publication on acceptance

- support for research data, including large and complex data types

- gold Open Access which fosters wider collaboration and increased citations

- maximum visibility for your research: over $100 \mathrm{M}$ website views per year

At BMC, research is always in progress.

Learn more biomedcentral.com/submissions 